\title{
Il viaggio italiano di Théophile Gautier
}

\section{Annalisa Bottacin}

\section{(2) OpenEdition}

\section{Journals}

\section{Edizione digitale}

URL: https://journals.openedition.org/studifrancesi/4537

DOI: 10.4000/studifrancesi. 4537

ISSN: 2421-5856

\section{Editore}

Rosenberg \& Sellier

\section{Edizione cartacea}

Data di pubblicazione: 1 avril 2012

Paginazione: 46-57

ISSN: 0039-2944

\section{Notizia bibliografica digitale}

Annalisa Bottacin, «ll viaggio italiano di Théophile Gautier», Studi Francesi [Online], 166 (I | LVI) | 2012,

online dal 30 novembre 2015, consultato il 19 novembre 2021. URL: http://journals.openedition.org/ studifrancesi/4537 ; DOI: https://doi.org/10.4000/studifrancesi.4537

\section{(c) (i) (9)}

Studi Francesi è distribuita con Licenza Creative Commons Attribuzione - Non commerciale - Non opere derivate 4.0 Internazionale. 


\section{Il viaggio italiano di Théophile Gautier}

\section{Premessa}

L'immersione nelle pagine di questo Voyage en Italie, da noi reperito quasi casualmente nella Biblioteca dell'Università di Ca' Foscari a Venezia, allorché eravamo impegnati intorno a una riflessione su alcune pagine stendhaliane del Journal, dedicate ai soggiorni di Henri Beyle nella Città dei Dogi, è avvenuta quasi spontaneamente, tramutandosi ben presto in un intento di traduzione, per altro fino allora mancante. Si tratta quindi, almeno in prima istanza, di un progetto sentimentale per le nostre origini veneziane - dato che gran parte del viaggio è dedicato a Venezia - progetto inerente a un testo la cui complessità delle argomentazioni ha sollecitato, accanto al mero esercizio di traduzione, una rigorosa ricerca nei labirinti delle biblioteche, altresì supportata da un'ampia serie di quesiti posti a specialisti, e ha richiesto da parte nostra uno sguardo attento alle minime tracce di percorsi già noti, ma non ancora interamente esplorati.

Tra i molti volumi della ricca Raccolta Fradeletto, donata all'Ateneo veneziano, allora denominato «Regio Istituto Superiore di Scienze Economiche e Commerciali», abbiamo reperito l'edizione del 1884, edita a Parigi da Charpentier, del Voyage en Italie. Italia ${ }^{1}$, che comprende anche i feuilletons dedicati a Firenze, città in cui la narrazione del périple italiano si arresta: delle visite a Roma e a Napoli la redazione dell'écrivain voyageur fu molto frammentaria. Una lettura stimolante che ci condusse verso una elaborazione delle varie edizioni, fino a scegliere quella di preferenza, edita da Marie-Hélène Girard nel $1997^{2}$.

L'immagine dell'Italia conosciuta da Gautier era quella proiettata sulla tela da tutti gli artisti che avevano visitato il nostro paese - molti vi si erano anche stabiliti - rendendo così immortale un luogo incantato per la bellezza incontaminata del suo paesaggio e per il fascino dell'antichità; non si era trattato per lo scrittore di un viaggio di formazione, secondo la connotazione originaria del Grand Tour, che aveva portato lungo le strade e le città italiane un gran numero di intellettuali, letterati, artisti e scienziati, in particolar modo nel Settecento.

Questa moda era ormai tramontata, e l'Italia veniva ora scoperta come paese. Un paese reso quasi interamente suddito da potenze straniere, un'Italia in costume d'Arlecchino, tanti erano gli stati in cui era divisa. E geograficamente con grandi diversità fra una terra e l'altra, fatto che si univa alle differenze tra i vari regimi. Gautier non si trova davanti a un paese omogeneo, il che viene sovente evidenziato al punto che l'esteta, di cui si avverte lungo tutto l'arco del racconto un profondo senso di rammarico per la drammatica situazione politica, è conscio di trovarsi in una terra dai profondi contrasti. Di conseguenza, i suoi reportages assumono in questo

(1) Th. Gautier, Voyage en Italie. Italia. Nouvelle édition considérablement augmentée, Paris, G. Charpentier et $C^{\text {ie }}$ Éditeurs, 1884.
(2) ID. Italia. Voyage en Italie, présenté et annoté par M.-H. GIRARD, Paris, La Boîte à Documents, 1997. 
momento - siamo nel 1850 ancora ben lontani dall'Unità d'Italia - una straordinaria importanza in quanto, almeno sino a Firenze, propongono una visione complessiva del Bel Paese. In essi Gautier pare imporsi quasi un'ossessiva ricerca di cose e fatti da narrare che stupiscano il lettore, rifugiandosi sempre più nel suo rêve d'art'.

Così l'Italia, paese infelice, si trasforma in un luogo in cui perdersi nel rêve, evadendo da un reale autentico piatto e a tratti perturbante. La religione del Bello, o meglio una continua ricerca di essa, domina questo primo viaggio italiano di Théophile Gautier, che si propone non solo di visitare quell'Italia romantica, a fosche tinte, immortalata nei drammi di Victor Hugo ma anche di descrivere momenti di vita quotidiana, feste popolari, ricorrenze, fatti di cronaca, cercando in essa nuove "patrie ideali" - quali Venezia in primo luogo, cui dedicherà la maggior parte dei capitoli del suo périple italiano, e dove si fermerà per ben cinque settimane, superando di gran lunga i pochi giorni di viaggio che lo condussero da Ginevra a Milano.

1. La descrizione del Tour italiano inizia dunque a Ginevra ${ }^{4}$. Gautier e l'amico Louis de Cormenin, già compagno di viaggio nel 1845 in Algeria, scelgono per entrare in Italia la via più diretta, quella del Valais e della strada del Sempione. Nessun accenno alle tappe precedenti in territorio francese. Fu un viaggio a lungo rimandato quello di Théophile Gautier verso l'Itala; comunque, in quel particolare momento il contesto politico del Bel Paese, dopo le sommosse del '48, interessava non poco i confinanti francesi, al punto che a Emile de Girardin' ${ }^{5}$, proprietario de La Presse, «quotidien auquel Gautier collabora pendant la moitié environ de sa vie professionnelle» ${ }^{6}$ e precisamente dal 1836 , venne l'idea di inviare un suo cronista per elaborare dei feuilletons che producessero un quadro più preciso della situazione italiana, seppur per lo scrittore, come ben rileva Marie-Hélène Girard,

al contesto politico si aggiungevano altresì forti motivi personali. In effetti Gautier aveva incontrato durante un viaggio a Londra nel 1849, l'affascinante Marie Mattei, [e la] prospettiva di raggiungerla a Venezia per qualche settimana l'indusse probabilmente al ritiro del passaporto alla fine di luglio del $1850^{7}$.

Il passaggio dalla Svizzera all'Italia si svolge in pochi giorni ma nel redattore è già forte la presa di coscienza del proprio lavoro, infatti

da Ginevra a Domodossola, a Milano, a Brescia, a Verona e finalmente a Venezia [...] i capitoli sposano i ritmi della diligenza o del treno, che diventa qui un nuovo referente letterario, decisamente assai moderno. Il tempo della narrazione è visibilmente regolato sul tempo del viaggio e ifeuilletons sono meno calibrati sulle distanze percorse e sulle fermate intermedie che non sullo spazio delle sei colonne canoniche a piè di pagina del quotidiano ${ }^{8}$.

(3) Cfr. R. JASINSKI, Les années romantiques de Théophile Gautier, Paris, Vuibert, 1929.

(4) «Nous avons bien peur-questo l'incipit della narrazione odeporica - d'avoir manqué notre premier pas sur la terre étrangère par un acte de paganisme, une libation au soleil levant!» (TH. GAUTIER, Viaggio in Italia (1852). Da Ginevra a Venezia. A cura di A. Bottacin. Pref. di M.-H. GiRARD. Testo francese a fronte, trad. di A. BotTACIN e S. Piserchio, note e revisione del testo di A. Bottacin e G. Zimmermann, Milano, La Vita Felice, $2010^{2}$, p. 22). La nostra precisa volontà di pubblicare questa prima traduzione italiana del Voyage en Italie in edizione bilingue ha indotto l'editore, per l'alto numero di pagine, a dividere l'opera in tre volumi: Da Ginevra a Venezia, Venezia, Da Venezia a Firenze. Tra l'altro nel 2011 è caduto il bicentenario della nascita di Gautier e questa prima traduzione italiana assume altresì il senso di doveroso omaggio.

(5) Cfr. P. Pellisier, Emile de Girardin, prince de la presse, Paris, Denoël, 1985.

(6) G. Bellati, Théophile Gautier journaliste à «La Presse». Point de vue sur une esthétique théâtrale, Torino-Paris, L'Harmattan Italia, 2008, p. 11.

(7) M.-H. GIRARD, Prefazione a Th. Gautier, Viaggio in Italia (1852). Da Ginevra a Venezia, cit., p. 7.

(8) Ivi, pp. 9-10. 
La prima importante tappa è Milano, città raggiunta via Briga, una volta superate le asperità del Sempione, ma il resoconto di viaggio inizia in Svizzera e precisamente a Ginevra; Gautier vede il percorso come una serie di "dagherrotipi", novità dell'epoca, nel duplice senso di un'amplificazione e di una proiezione. E sempre il critico alla ricerca di continue rivelazioni, che non viene a promuovere solo una mera descrizione di cose viste, più o meno piacevoli e arricchenti, dai contorni ben definiti. L'esplorazione dei luoghi, scoperti per la prima volta, si muove all'interno di un immaginario che non intende creare o produrre nuove aperture, ma rinvia di continuo a percorsi virtuali, prodotti dalla propria mente, che si scontrano con l'oggetto che gli appare davanti e che a volte può anche venire rifiutato.

Ne consegue un'ambiguità che rimane irrisolta ma che si traduce in quella che è l'opera del critico, alla ricerca di un ordine che alla fine del viaggio non appare ritrovato, ma il fluire della vita italiana e l'accumularsi degli eventi, in un paese anelante la libertà, creano una trama, nella visione data da Gautier, che ancor'oggi, a una moderna lettura, situa questa narrazione tra i più bei resoconti di un viaggiatore in Italia, in una quête, a volte sublimatoria a volte inquietante, nei sentieri enigmatici del pittoresque. Il passaggio verso l'Italia avviene attraverso la strada del Sempione, da dove, scrive Gautier,

[u]ne diligence devait nous conduire à Milan en passant par le Simplon, non pas la même, car on en change presque à chaque territoire qu'on traverse, le gouvernement ayant le monopole des transports, et nous n'avions d'autre souci à prendre que de nous laisser transvaser d'une voiture genevoise dans une voiture savoyarde, qui nous céderait à une voiture suisse, laquelle nous transmettrait à une voiture piémontaise qui nous verserait dans une malle autrichienne?.

Si avverte sin d'ora quanto l'immagine dell'Italia sia soggetta a forme di controllo burocratico, dovuto ai differenti governi, e quanto questo sconvolgimento dell'ordine indispettisca il viaggiatore, che subito percepisce la rottura di un equilibrio essenziale per chi si metta in viaggio - con le conseguenze nell'ambito dell'estetica che derivano da questo o da quel regime nella propria organizzazione sociale. Gautier, in effetti, e questo lo si riscontra anche nel Voyage en Italie, giudica un sistema politico dalle proprie scelte artistiche, e ciò lo infastidisce più ancora del volto rattristato degli individui che incontrerà lungo il suo cammino. Perché libertà è sinonimo di atto creativo, che inevitabilmente conduce all'attuazione della Bellezza.

Al di là del Sempione, nota Gautier, «[o]n n’est plus séparé que par une crête de montagnes de cette Italie, dont le nom est si puissant, selon Henri Heine, qu'il fait chanter Tirily, même à un philistin berlinois» ${ }^{10}$; le mille asperità del cammino, a tratti molto difficoltoso, si traducono in uno spettacolo «d'une sauvagerie tout à fait romantique» ${ }^{11}$ che lo incanta; gli orridi, i fiumi tumultuosi, le cascate, le nevi, l'aspra vegetazione, che ci riportano alla mente alcuni paesaggi montani di Francesco Foschi ${ }^{12}$, assumono il significato simbolico di una nuova dimensione, producendo nell'artista un effetto di straniamento che lo induce a considerare il brutto quale parte del bello

(9) Th. Gautier, Viaggio in Italia (1852). Da Ginevra a Venezia, cit., pp. 60-62.

(10) Ivi, p.72.

(11) Ibid.

(12) Alludiamo in particolar modo alle gran-

di tele del settecentesco manierista anconetano, raffinato paesaggista diventato celebre per i suoi suggestivi paesaggi innevati», quali ad esempio il Paysage montagneux sous la neige avec diligence e il Paysage avec rochers et voyageurs (1805) del Musée de Grenoble. 
nella sua connotazione di pittoresque ${ }^{13}$. Le selvagge e tortuose Gole di Gondo segnano il punto più pericoloso del passaggio:

A peu près vers cet endroit le Frasinone et deux torrents qui viennent des glaciers du Rosboden, se précipitent dans l'abîme avec une fureur et un bruit épouvantables. La route suit une corniche en saillie sur le gouffre [...]. En bas est la nuit, le froid, la mort; jamais un rayon de soleil n'arrive jusque-là. C'est l'endroit le plus farouchement pittoresque du passage ${ }^{14}$.

Attraverso questa scompigliata natura la strada prosegue; superato il ponte di Crevola «qui termine avec une merveille tous les prodiges du Simplon», che chiude la valle di Domodossola, ecco finalmente l'Italia, una nazione che si presenta ai suoi occhi in modo inatteso.

Au lieu du ciel d'azur, des tons orangés et chauds que nous rêvions, sans penser après tout que l'Italie du nord ne peut avoir le climat de Naples, nous trouvions un ciel nuageux, des montagnes vaporeuses, des perspectives baignées de brumes bleuâtres, un site d'Ecosse lavé par un aquarelliste anglais, un paysage humide, verdoyant, velouté, digne d'être chanté par un poète lakiste ${ }^{15}$.

L'arrivo nel Bel Paese è caratterizzato da una pioggia incessante, particolare più di una volta evidenziato da Gautier che non nasconde un senso di stupore, data la stagione estiva, considerando che il viaggio da Ginevra a Venezia ha luogo dal 4 al 10 agosto 1850. Nel primo impatto con l'Italia, a Domodossola, ha come interlocutore il cameriere della locanda, al quale viene chiesto se vi sia in quel luogo un teatro, per ammazzare la serata.

Si evidenzia subito, invero, che tendenza essenziale in questo périple, non sarà la curiosità di tessere relazioni intersoggettive - come ad esempio era avvenuto per Stendhal - non vi è partecipazione. Manca la forma dell'incontro. Molte serate italiane di Gautier e Cormenin si svolgeranno nelle sale da spettacolo, che in molti casi è un modo di passare il tempo in un paese dove l'esteta è unicamente profeta ispirato dalla sua Bellezza; certo è che Gautier non intendeva cambiare il mondo né per altro sarebbe stato possibile; comunque l'Italia, immersa in uno stato confusionale imperante, poteva difficilmente essere interpretata altrimenti da uno straniero che non l'aveva mai visitata. È un senso di smarrimento quello che cogliamo a volte in Gautier, non un senso di distacco, quasi un'impotenza, evidenziata in certe frasi, che una più approfondita lettura rende manifeste. Per altro al lettore d'oltralpe, diretto fruitore dei feuilletons, pur simpatizzando per gli insorti e i martiri dei recenti moti rivoluzionari, i problemi politici italiani passavano in secondo piano dinanzi alle descrizioni paesaggistiche, architettoniche e monumentali della penisola, e Gautier questo ben lo sapeva.

Come tuttavia non formulare un'ulteriore ipotesi, che ciò sia anche dovuto al fatto di non sentirsi a proprio agio in spazi in cui la parola è negata, producendo atteggiamenti che sovente possono sviluppare incomprensioni e tensioni manifeste? Ecco che il teatro, luogo del falso per eccellenza e luogo circoscritto, volutamente lontano dalla vita reale perché deve divertire o commuovere o decostruire, ha un ruolo fondamentale in questo Voyage, quasi che attraverso le scenografie e i ruoli degli attori fosse possibile addentrarsi nella realtà italiana, non solo per perdersi nel sogno

(13) Cfr. K. Rosenkranz, Estetica del Brutto. A cura di R. BoDEI, Bologna, Il Mulino, 1984.

(14) Th. Gautier, Viaggio in Italia (1852). Da
Ginevra a Venezia, cit., p. 98.

(15) Ivi, p. 104. 
bensì per slittare in esso. Poco importa se a Domodossola il teatro è chiuso - data la stagione estiva questo avverrà più di una volta - basta una troupe di burattinai, quella di Luciano Zane, cui viene chiesto di improvvisare uno spettacolino per i due stranieri per rendere gradevole la serata. Appena si sparge la voce della rappresentazione, non manca di arrivare gioiosa «la marmaille de la ville, que nous avions dit de laisser entrer, [...] et c'était plaisir de voir pétiller ces yeux noirs et rire ces jolies bouches roses» ${ }^{16}$. Lo spettacolo produce un piacevole effetto, che ben sminuisce il triste impatto con la realtà esterna: «Nous revenions très-enchantés de Luciano Zane, qui écrit lui-même ses pièces, peint ses décorations, modèle et habille ses marionnettes ${ }^{17}{ }^{17}$, e una volta in diligenza verso Milano «tout en roulant dans l'obscurité, nous rêvions toujours aux belles marionnettes, qui faisaient des gestes extravagants et cabriolaient à travers notre sommeill» ${ }^{18}$. Tramutato in oggetto onirico, il teatro cattura e sublima.

Lasciata Domodossola, Gautier e Cormenin giungono all'alba «sur les bords du lac Majeur, à la hauteur de Baveno»; l'acqua ingrossata dalle piogge e dai venti «ondulait assez fortement, et le lac se donnait des airs de houle comme la mer» ${ }^{19}$. Il tempo pareva lentamente rasserenarsi, malgrado gli oscuri nuvoloni ammassati sopra le alture che circondano il lago, cosicché «le lac Majeur que nous nous étions figuré comme une coupe d'or remplie d'azur, avait une mine tempétueuse et mâle. Nous trouvions la beauté où nous attendions la grâce $\gg^{20}$. Sulle terrazze, che scendono a gradoni verso il lago, lo scrittore rimane stupito di fronte a quella che definisce une «bizarrerie», scorgendo alcuni cespugli di ortensie gigantesche, prive di quella sfumatura rosa o mauve che poteva ammirare in Francia, ma che, al contrario, «offraient des teintes d'un azur charmant: ces hortensias bleus - nota ancora Gautier - nous ont beaucoup frappé, car le bleu est la chimère des horticulteurs $\gg^{21}$, e ne rivela il segreto dovuto alla loro crescita nella terra della brughiera o cospargendo il terreno di soda.

Dalla riva scorge le Isole Borromee, dove «la maçonnerie y joue un grand rôle, comme dans presque tous les jardins italiens, qui sont plutôt des morceaux d'architecture que des jardins ${ }^{22}$, supera Arona e Sesto Calende dove il lago finisce - l'altra sponda è piemontese - e inizia la dominazione austriaca; in effetti i due viaggiatori non tardano a vedere per la prima volta «les pantalons bleus collants et la tunique blanche des Hongrois», visione per altro ricorrente per buona parte del tragitto.

«Gallarate et Rho vous amènent à Milan en deux relais» ${ }^{23}$, questo il nuovo passaggio. La magnificenza di una grande città già viene a percepirsi dal lungo viale che conduce all'Arco della Pace, «[u]n arc de triomphe à qui celui du Carrousel passerait entre les jambes, et qui pourrait lutter de grandeur avec l'arc de l'Étoile» ${ }^{24}$, che si staglia, con i due caselli daziari, al centro del piazzale che funge da testata alla strada del Sempione, creando un effetto monumentale. Sullo sfondo s'intravede ormai il Castello Sforzesco e poco oltre lo sguardo viene carpito dalla bellezza maestosa del Duomo, cui sarà dedicata una lunga e dettagliata descrizione. Lo scrittore pare investito da nuove sensazioni, più positive, meno angoscianti; in effetti la frase si distende, concretizzando, forse per la prima volta dall'arrivo in Italia, il piacere intenso dell'esplorazione e della scoperta: «on sent, à l'élévation des bâtiments, un mouvement de la population, à la propreté et à la confortabilité générales, qu'on est dans une capitale vivante, chose rare en Italie, où il y a tant de villes mortes ${ }^{25}$. A Milano dedicherà due feuilletons.

(16) Ivi, p. 110.

(17) Ivi, p. 116

(18) Ivi, p. 118.

(19) Ivi, p.132.

(20) Ibid.
(21) Ivi, p. 134.

(22) Ivi, p. 136.

(23) Ivi, p. 148.

(24) Ivi, pp. 148-150.

(25) Ivi, p. 150. 
Visitata frettolosamente Brescia e giunti a Verona, «[d]e Brescia à Vérone nous n'avons pas grand-chose à mentionner ${ }^{26}$, questo il rapido commento, e compiuto il breve giro turistico alla Tomba di Giulietta, alle Arche scaligere e all'Arena, Gautier e Cormenin si recano alla stazione ferroviaria, per prendere il treno che li condurrà a Venezia. Dall'ottobre del 1849 erano infatti state organizzate tre corse giornaliere da Verona Porta Vescovo a Marano di Mestre, cui seguiva il passaggio in gondola per Venezia, in quanto il ponte translagunare venne inaugurato il 29 giugno 1850. Gautier fu, invero, tra i primi ad attraversarlo e giungere così fino a Venezia, in circa quattro ore con fermate intermedie, a bordo della locomotiva ${ }^{27}$.

2. La parte centrale del viaggio ha inizio ora. È la Venezia notturna che appare a colui che sogna di essere un veneziano ai tempi di Casanova; l'arrivo nella città d'acqua si trasforma subito in un «voyage dans le noir», paragonabile a un sogno pauroso in notti popolate da incubi, «sur les ailes de chauve-souris de Smarra» ${ }^{28}$. Il desiderio di visitare la città dei Dogi, a lungo agognata, si trasforma, al passaggio dal treno alla gondola che condurrà Gautier all'Hôtel de l'Europe $e^{29}$, posizionato all'imbocco del Canal Grande, in inquietante ricognizione di un topos mitico, nel contempo attrattivo e repulsivo.

La Serenissima, nella plumbea notte lagunare, scorta dal treno in corsa tra lividi lampi e scrosci di pioggia, al punto che lo stesso convoglio «semblait chevaucher à travers le vide comme l'hippogriffe d'un cauchemar $»^{30}$, impedendo di differenziare il cielo dal mare, produce una visione fantastica agli occhi dei due viaggiatori; il paesaggio étrange viene ancora una volta interpretato quale presenza fantomatica di revenants, cosicché, nota l'écrivain voyageur, «[n] ous croyons circuler dans un roman de Maturin, de Lewis ou d'Anne Radcliff, illustré par Goya, Piranèse et Rembrandt» ${ }^{31}$, innescando meccanismi che non erano certo estranei a Gautier, la cui ceuvre fantastique iniziò a prodursi nel 1831 con La cafetière (è anche autore di una recensione del Diable amoureux di Jacques Cazotte apparsa su «La Presse» il 12 maggio 1845), fino a quel capolavoro del genere, quasi un testamento spirituale ed estetico, che fu Spirite, redatto nel $1865^{32}$.

Da questa proiezione della città escono pagine che la confusione demoniaca dell'arrivo rende palpitantes; Gautier è il flâneur che intende trasmettere anche l'evento improbabile, intraprendendo un racconto che non sarà solo una mera descrizione di monumenti e dettagli di vita. Il flâneur sta in ascolto, in attesa della scoperta, dell'avvenimento. Il coinvolgimento si farà sempre più forte, perché accade che egli si trovi ad attraversare spazi déjà vus, precedentemente visitati in un rêve prémonitoire, in scenografie prodotte dalla sua mente, «dans la chambre noire du cerveau» ${ }^{33}$. La

(26) Ivi, p. 210.

(27) Cfr. A. Bernardello, La prima ferrovia fra Venezia e Milano. Storia della Imperial-Regia Privilegiata Strada Ferrata Ferdinandea Lombardo-Veneta (1835-1852), Venezia, Istituto Veneto di Scienze, Lettere ed Arti, 1996.

(28) Th. Gautier, Viaggio in Italia (1852). Da Ginevra a Venezia, cit. p. 238.

(29) Ora sede della Biennale. Vi è una targa che ricorda il soggiorno di Gautier.

(30) Ivi, p. 236.

(31) Ivi, p. 242.

(32) Cfr. J. CazotTe, Le Diable amoureux (1776).

Texte établi, annoté et présenté par A. BotTACin, Milano, Cisalpino-Goliardica, 1983; ID., Il profilo del diavolo. Itinerario nella simbologia e nel fantastico del "Diable amoureux" di Jacques Cazotte, Este, Zielo, 1984; ID., Venezia 'topos' fantastico e inquietante nel "Voyage en Italie" di Théophile Gautier in «I Quaderni del CRIER», n. 2, 1997, pp. 67-81; ID., Un esteta attratto dal visionario: Théophile Gautier lettore di Jacques Cazotte in «La Questione Romantica» n. 3/4, 1997, pp. 87-111. Anche il romanzo di Cazotte si svolge per gran parte a Venezia.

(33) Sulla flânerie, cfr. W. BENJAMIN, Di alcuni motivi in Baudelaire in Angelus Novus. Saggi e frammenti, Torino, Einaudi, 1962, pp. 87-126; G. Nuvolati, Lo sguardo vagabondo. Il flâneur e la città da Baudelaire ai postmoderni, Bologna, Il Mulino, 2006. 
ville intuitive, luogo del desiderio, viene così a sovrapporsi nelle forme a quella reale, tanto è grande la forza del rêve e l'investimento diventa totale, allorché l'immagine virtuale si concretizza.

$\mathrm{Al}$ mattino, quel paesaggio configurato come enigma si rivela dal balcone della stanza dell'albergo in tutta la sua maestosa bellezza: ciò che si presentava allo sguardo «était un tableau ravissant, - rivela Gautier - aussi clair que celui de la veille était sombre ${ }^{34}$, dall'effetto di un'opera d'arte degna del più valido pittore, costituito dalla Dogana da Mare, dalla Chiesa della Salute, che riconosce «sur-le-champ [...] d'après le beau tableau de Canaletto, qui est au Musée»> ${ }^{35}$, dalle isole della Giudecca e di San Giorgio Maggiore, da una scuola di nuoto sita all'inizio del canale ${ }^{36}$, e da un gran numero di barche di varie tipologie, alcune coloratissime, che portavano le merci in città dalle isole circostanti e dalla terraferma.

Nous avons indiqué - continua ancora Gautier - le plus exactement qu'il nous a été possible, les principaux linéaments du tableau; mais ce qu'il faudrait rendre, c'est l'effet, c'est la couleur, c'est le mouvement, c'est le frisson de l'air et de l'eau, c'est la vie ${ }^{37}$.

Finalmente a suo agio nell'"evento", di cui viene ad appropriarsi con sempre maggiore forza, l'esteta è appagato; Venezia, altrove assoluto, rinvia a una sacralità che gli è propria, che egli assapora e condivide; il luogo in cui l'instancabile ricercatore del Bello viene finalmente a posarsi per riprendere il respiro. La ville intuitive, patria d'elezione, gli è di fronte, forse in alcuni tratti un po' discordante da quella a lungo desiata; in ogni caso è certo che il distacco da essa sarà profondamente traumatico:

La saison s'avançait. Notre séjour à Venise s'était prolongé au-delà des limites que nous lui avions fixées dans le plan général de notre voyage. Nous retardions notre départ de semaine en semaine, de jour en jour, et nous trouvions toujours quelque bonne raison pour rester ${ }^{38}$.

Negli ultimi giorni tale esaltazione si trasforma in «véritable fièvre» ${ }^{39}$; la città è attraversata a passo di corsa in un'estrema appropriazione che induce a rivisitare gran parte dei luoghi scoperti e riscoperti, e non mancano neppure i giri in gondola, il cui remo è affidato al giovane Antonio, lungo i canali e sullo specchio lagunare.

Gautier, scrittore brillante e fantasioso, da pittore qual era, è indotto a rendere palpabili gli effetti della luce e delle prospettive veneziane in continui giochi di forme, di linee, di rilievi e di colori. Le sua pagine escono spesso da immagini che la sua arte elabora in simbologie; spesso è ispirato da una tela, da un acquarello, da un profilo di palazzo, da un angolo di chiesa, dall'ellisse di una cupola. Alcune pagine veneziane sono cesellate da un orafo raffinatissimo; la ricerca della parola è supportata da una cura meticolosa: spesso anche la fanciulla, scorta per strada, diventa l'immagine

(34) Th. Gautier, Viaggio in Italia (1852). Da Ginevra a Venezia, cit., p. 248.

(35) Gautier allude al dipinto la Chiesa della Salute, acquistato dal Louvre nel 1818, che non era opera di Canaletto bensì di Michele Marieschi, uno dei più temibili concorrenti del vedutista veneziano. Egli riusciva a strappare a Canaletto molti importanti clienti, abbassando drasticamente i prezzi delle sue tele. Morì a soli 32 anni e solo allora Canaletto provò un certo sollievo. Ma non fu il solo; come dimenticare Luca Carlevarijs e il nipote Bernardo Bellotto?

(36) I primi stabilimenti balneari a Venezia nac- quero come galleggianti, ormeggiati a poca distanza dalla città, con lunghe file di camerini e di servizi disposti su grandi chiatte affacciate sull'acqua. Il primo, su imitazione di quello triestino, fu il «Grande Stabilimento Galleggiante» del dottor Tommaso Rima realizzato nel 1833 e posto di fronte alla Dogana, direttamente sul Bacino di San Marco.

(37) Ivi, p. 260.

(38) Th. Gautier, Viaggio in Italia. Venezia, a cura di A. BotTacin, Milano, La Vita Felice, 2010, p. 648 .

(39) Ivi, p. 654. 
di un quadro o di una statua ${ }^{40}$. Specie nei feuilletons dedicati a Venezia come a Firenze, vi è una trasposizione continua dell'emozione. In particolar modo a Venezia ritrova quell'Oriente già assaporato in Spagna che lo avvince ${ }^{41}$, in totale esaltazione del primato dell'estetica, intendendo per estetica l'attività dell'artista che organizza il mondo della sensibilità e si propone di dare un'ipotesi concreta a questo mondo, nel culto della religione del Bello che è ricerca stessa del divino e glorificazione di esso. Dopo i sei primi capitoli dedicati all'inizio del viaggio, dal settimo al ventiseiesimo viene delineata Venezia. Se ne aggiungeranno poi altri tre, uno dedicato a Padova, un altro a Ferrara e l'ultimo, più corposo, a Firenze.

Il panorama completo di Venezia viene scorto per la prima volta dal Campanile di San Marco (rigorosamente sempre scritto con lettera maiuscola ed in italiano quasi a evidenziarne l'unicità); a grandi linee Gautier elenca già quelle che saranno le cose da vedere, per lo più riportate dalle guide, ma con qualche eccezione, come «San Servolo, où est l'hôpital des fous», che visiterà e descriverà intensamente nel capitolo XXI, un luogo da cui resta molto impressionato, e che innesta una riflessione produttrice di immagini perturbanti: «le moine de San-Servolo, les figures des fous et les monstres fantastiques de la fresque se combinaient dans un cauchemar extravagant et sombre comme un roman de Lewis ou de Mathurin ${ }^{42}$.

La descrizione della città d'acqua inizia dalla Chiesa di San Marco, quindi Palazzo Ducale, che sarà, al pari della basilica, minuziosamente narrato, il Canal Grande «la plus merveilleuse chose du monde ${ }^{43}$ con i suoi palazzi, eretti da grandi nomi dell'architettura. Alcuni sono in totale stato di decadimento, al pari dell'aristocrazia veneziana; altri sono di proprietà di stranieri, come la Ca' d'Oro, «un des plus charmants du grand

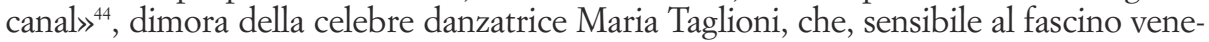
ziano, «en a plusieurs en pension qu'elle entretient par pure commisération pour leur beauté; on nous en a signalé trois ou quatre à qui elle fait cette charité de réparations» ${ }^{45}$.

Palazzo Vendramin Calergi, nella zona di San Marcuola, ospitava la duchessa de Berry, Maria Carolina di Borbone-Napoli, che Gautier ricorda più di una volta anche per la generosa elargizione di una delle lanterne che illuminano il traghetto della Piazzetta, che è ancor oggi «orné de lanternes gothiques, historiées de figures de saints, plantées sur des poteaux qui trempent dans la mer» ${ }^{46}$. Palazzo Mocenigo aveva ospitato Lord Byron durante il soggiorno veneziano del 1818 e Palazzo Labia, a San Geremia, con affreschi del Tiepolo, era proprietà di nobili spagnoli, appunto i Labia, da cui aveva preso il nome. Palazzo Barbarigo era noto per alcune tele molto celebri di Tiziano, che i due amici avevano incluso tra le cose salienti da vedere; sfortunatamente il console di Russia le aveva appena acquistate per lo zar e la preziosa collezione era già stata sigillata ed era pronta per la spedizione. Pur in mancanza di tali preziosità, Gautier e Cormenin visitano il palazzo dove restano alcuni quadri di poco valore, e rimangono colpiti dai soffitti a cassettoni dorati «dans un fâcheux état de dégradation». Tuttavia, ciò che più impressiona è un oggetto di grande bellezza, «un berceau merveilleux surchargé d'une ornementation extravagante et d'une richesse folle comme le berceau d'un fils du roi longtemps attendu $\gg^{47}$, costruito per il

(40) Questo clima di deliziosa nonchalance, richiama alla mente, pur in un contesto déco, la Venezia sognata di G. BARBIER in alcune sue opere quali ad esempio Le nozze del Doge con l'Adriatico, En bateau, Casanova o nei disegnini che illustrano l'articolo di A. FLARMEnT, Les verres de Venise, apparso sulla «Gazette de Bon Ton» del 1913. (Cfr. G. BARBIER, La nascita del déco, a cura di B. Martorelli, Venezia, Marsilio, 2009).

(41) Cfr. M.P. Pedani, Venezia Porta d'Oriente,
Bologna, Il Mulino, 2010.

(42) Th. Gautier, Viaggio in Italia. Venezia, cit., p. 528.

(43) Ivi, p. 180.

(44) Ivi, p. 190.

(45) Ivi, p. 194.

(46) Th. Gautier, Viaggio in Italia (1852). Da

Ginevra a Venezia, cit., p. 262. p. 614 . 
primogenito della famiglia Barbarigo. Ora la culla è vuota, i quadri di Tiziano sono sulla via delle Russie, la pioggia filtra dalle dorature dei soffitti e anche la facciata ammuffita e verdastra sembra cadere nel Canal Grande. Il ritratto della decadenza delle grandi famiglie veneziane è specchio di una città che ha perduto ogni splendore, il che colpisce profondamente Gautier, tanto da lasciare il palazzo «le cœur navré. Rien n'était triste - prosegue lo scrittore - comme ce berceau d'une famille éteinte dans un palais croulant $\gg^{48}$.

Sono quattro i capitoli dedicati alla vita che si svolge a Venezia, e Gautier non manca sovente di sottolineare l'estremo disagio della popolazione per la dominazione asburgica: vi sono intere pagine in tema, che ricordano ad esempio, con sottile ironia mista a sincero malessere, la caduta delle bombe aerostatiche sulla città durante l'assedio o l'esecuzione della musica tedesca in piazza o ancora le solennità per la festa dell'imperatore Francesco Giuseppe, dalle cui cannonate, iniziate all'alba, i due turisti vengono svegliati bruscamente, impauriti per il possibile inizio di un nuovo attacco bellico, o lo sventolio della bandiera austriaca sul pilo di Piazza San Marco, dove fino poco tempo prima si ergeva, superbo, il vessillo della Serenissima.

Il soggiorno veneziano sta giungendo al termine. La magia di Venezia, che si era così ben accordata con l'incontro d'amore con Marie Mattei, quasi a farne sublime sfondo, lentamente si dileguava, con il brusco passaggio dalla gondola al treno, nel cammino verso Firenze, dissolvendosi lentamente.

Quanto è drammatico, invero, allontanarsi da una città unica, oggetto di affezione, descritta in ogni sua parte, trasposta da quella onirica nelle sue forme materiali, e ridisegnata dalle ricognizioni dello sguardo in venti capitoli, che narrano di chiese, di palazzi, di sale da teatro, di Belle Arti, di attimi di vita lagunare, di isole e isolette. Flâneur attento e incantato, sempre accompagnato dal fedele Cormenin, Gautier è colto sino alla fine da un indefinibile impulso di spostarsi da un sito all'altro, tra caffè e piccole osterie come quella, più volte menzionata, di Quintavalle del signor Zuane, sotto il fresco pergolato di verdi pampini e foglie di fico, a degustare la famosa «soupe aux pidocchi» ${ }^{49}$. L'esteta continua sempre più a percepire, nell'esperienza della quotidianità, profonde analogie con quel topos fatato finalmente raggiunto, in una poursuite che è estremo omaggio alla Bellezza.

3. Il viaggio che condurrà Gautier a Firenze, inizia dunque in treno. La strada ferrata si arrestava a Padova, con soste a Mestre, Marano, Dolo, Ponte di Brenta. Una volta costeggiate le mura della città, il treno entrava in stazione nei pressi di Porta Codalunga, e di lì veniva raggiunto con i cavalli il centro cittadino.

La fase conclusiva di questo périple italiano è narrata in soli tre capitoli, forse per mancanza di tempo o di energia e, come rileva Girard, «si contraddistingue dalla prima sia per le circostanze biografiche che per le condizioni di pubblicazione» ${ }^{50}$. In effetti, la redazione di questo carnet de voyage fu quanto mai complessa: Gautier aveva promesso il regolare invio a «La Presse» dei pezzi che Girardin aveva già lautamente onorato. In realtà egli redasse e spedì i primi feuilletons che sarebbero apparsi dal 24 settembre al 15 ottobre 1850, in attesa di redigere i primi dodici capitoli di Italia, titolo del volume edito nel 1852 da Victor Lecou. Come rileva ancora Girard, pare che dalla tappa fiorentina lo scrittore abbia rinunciato a inviare i suoi itinerari italiani, rimandando il tutto al ritorno in sede; comunque le tredici puntante mancanti iniziarono ad apparire solo a partire dal 12 settembre 1851.

(48) Ibid.

(49) Ivi, p, 262.
(50) Th. Gautier, Viaggio In Italia. Da Venezia A Firenze, a cura di A. BotTacin, Milano, La Vita Felice, 2010, p. 7. 
Padova è dunque la prima tappa del nuovo percorso; una volta lasciata la stazione i due viaggiatori giungono all'albergo in calesse, disabituati dalla quiete veneziana allo scalpitio dei cavalli e agli usuali rumori del traffico cittadino. Si tratta di un breve soggiorno che comunque non esclude la visita ai principali monumenti, alle chiese più note e all'antica Università; non manca nemmeno una sosta allo storico Caffè Pedrocchi, dove si recano dopo cena, «célèbre dans toute l'Italie pour sa magnificence», che viene accuratamente descritto e riporta alla mente di Gautier lo stile di Percier e Fontaine per «piliers... colonnes...palmettes...» $\rangle^{51}$.

La serata si conclude ancora una volta a teatro, dove veniva rappresentato il Barbiere di Siviglia di Rossini e un balletto locale, la cui scenografia, in un contesto di briganti, di fanciulle impaurite, di viaggiatori sperduti nei boschi, il tutto sorretto da un insieme di danze e cruenti combattimenti, rammenta a questo appassionato del balletto i «temps les plus glorieux des Funamboles» ${ }^{52}$. Questa è l'ultima tappa nel Lombardo-Veneto, un regno «à qui rien ne manque, hélas! sinon la liberté»\$3 .

Un omnibus conduce l'écrivain voyageur a Rovigo, una volta attraversato il Po che gli ricorda vagamente il Guadalquivir vicino a Siviglia. Dopo la visita abbastanza discreta della dogana ai loro bagagli, Gautier e il compagno risalgono in diligenza dove i posti migliori erano occupati da due preti, che, a dire dello scrittore, nello Stato della Chiesa hanno uno smisurato potere, «ayant à la fois le ciel et la terre, les clefs de l'autre monde et de celui-ci, pouvant vous damner et vous faire pendre, tuer votre âme et votre corps» ${ }^{54}$.

Quella triste compagnia, che faceva apparentare la diligenza a un carro funebre, li accompagna sino a Ferrara, dove il ricordo del Tasso e dell'Ariosto sono vivissimi, ma la breve visita alla città è turbata da una figura orrenda di questuante, che riporta alla mente i personaggi spettrali di Zurbarán, forse un penitente della Confraternita della Morte, tristo accompagnatore dei condannati al patibolo che negli Stati Pontifici, fatto ben evidenziato da Gautier, erano molto numerosi e strenuamente puniti per le loro idee liberali, per altro assai estranee a problemi di fede.

La visita a Bologna è caratterizzata dalla speranza di un incontro con Gioacchino Rossini, il che non avviene per la presunta assenza del maestro; lo scrittore è assai colpito dalla pendenza delle due torri, che vengono osservate con una certa titubanza per un possibile crollo. Il senso di profondo malessere, che ha ispirato queste pagine, verrà meno solo in Toscana, governata dagli Asburgo-Lorena, noti per il loro liberalismo moderato.

In un clima più disteso, anche la scrittura pare rasserenarsi e la parola viene sempre più spesso a tradursi in immagine, in contesti meno drammatici: l'esplorazione dello spazio, certo pur limitata alla descrizione delle architetture fiorentine, è ricca d'intuizioni quanto mai interessanti. Quei possibili segni di estraneità, qua e là percepibili nei due capitoli precedenti, dedicati a Padova e a Ferrara, si esauriscono per dare vita alla descrizione di una città viva, in cui certi luoghi funzionano nuovamente da tracciati utopici dalla grande forza seduttiva.

L'oggetto veneziano, la città incantata, «l'Armide de l'Adriatique» ${ }^{55}$ è ancora viva rimembranza; essa aveva sottratto Gautier a una partenza che si era prolungata ben oltre i termini previsti, imponendosi negativamente sulle finalità del programma di marcia e limitando i giorni di sosta nelle altre città.

(51) Ivi, p. 32

(52) Ivi, p. 34. (Cfr. F. BRunet, Théophile Gautier et la danse, Paris, Champion, 2010).
(53) Ivi, p. 46.

(54) Ivi, p. 62.

(55) Ivi, p. 98. 
L'andare in cerca del nuovo da scoprire, comunque, riprende corpo e s'insinua nella pagina sin dall'inizio del capitolo che ha per titolo Florence, al punto che il lettore percepisce di netto che potrà nuovamente ritrovare le personalissime trouvailles gautieriane dal tratto sublime, ammalianti campi di visualizzazione di spazi prediletti.

Sul versante dell'aneddotico, in effetti, si articola la descrizione del passaggio montagnoso da Bologna a Firenze, che apre il ventinovesimo e ultimo capitolo del Voyage en Italie. Il ventoso Appennino richiama un'escursione in Cabilia in un paesaggio ben diverso, durante il viaggio compiuto in Algeria nell'estate del 1845, tra «montagnes dorées par le soleil d'Afrique» ${ }^{56}$; le alture «médiocres», prive d'alberi, sono battute dalla violenza dei venti che rendono la temperatura molto rigida. A tal proposito, lo scrittore elogia il Granduca Leopoldo, il quale «a fait, au point culminant de la route, élever un mur de pierre pour protéger les voyageurs contre ces rafales glacées qui les transiraient et les renversaient» ${ }^{57}$.

Il viaggio prosegue con un nuovo controllo doganale e il paesaggio toscano, che lentamente appare alla vista, ha una dolcezza da far presumere di trovarsi finalmente vicino a una «grande ville vivante, chose rare en Italie, cet ossuaire des villes mortes ${ }^{58}$. E morte è nuovamente sinonimo di oppressione e di annientamento di ogni forma di libertà.

Giunti a Porta San Gallo, la porta urbica settentrionale, Gautier e Cormenin si vedono costretti ad attendere più di un'ora per le formalità di polizia; finalmente alzata la sbarra di legno entrano in città e

la voiture put rouler sur le pavé cyclopéen de Florence. Nous disons cyclopéen parce que, comme les murs qui portent ce nom, il est composé de pierres de figures inégales, s'agençant par les angles, aussi que des morceaux de casse-tête chinois ${ }^{59}$.

Tuttavia, anche in quel luogo armonioso, i due viaggiatori, di primo impatto, vengono profondamente colpiti da «une apparition [...] effrayante»; scorgono infatti due file di «spectres noirs masqués» ${ }^{60}$, appartenenti alla Confraternita dei Penitenti, che stavano scortando un funerale. Tale spettacolo inatteso, apparentemente foriero di sciagura, induce a una lunga digressione sul senso religioso che regna in Italia; solo l'arrivo all'Hôtel de New York, per altro abbastanza confortevole, dissolve i turpi pensieri e ha la funzione d'immettere questo voyageur, sovente turbato in fondi d'ombra, nella vera dimensione della città. La Firenze rinascimentale lo avvince con la bellezza delle sue forme e non stupisce che il teorico de l'art pour l'art la preferisca alla Roma barocca, di cui lascia sulla pagina ben poche tracce; con uno stile che definiremmo lumineux, il prosateur-poète, ironico e fantasioso, continua a offrire al lettore le sue evocazioni penetranti e fedeli, in un luogo che condivide pienamente. Dalla Loggia dei Lanzi a Piazza della Signoria, a Palazzo Vecchio, alle passeggiate sul Lungarno attraverso gli antichi ponti, tutto è gioia per lo sguardo. Gli è altresì gradito riportare l'episodio del crollo del Ponte alla Carraia narrato nella Cronaca dal Villani:

Au mois de mai 1504, une bizarre annonce répandue dans Florence faisait savoir aux habitants «que ceux qui désiraient avoir des nouvelles de l'autre monde n'avaient qu'à se rendre sur le pont alla Carraia» ${ }^{61}$.

L'assembramento per assistere alla rappresentazione dell'inferno fu tale da procurare il disfacimento del ponte, che trascinò con sé in acqua tra le fiamme molte 
persone che, come prometteva l'annuncio, ebbero «des nouvelles directes de l'autre monde en allant les chercher eux-mêmes» ${ }^{62}$.

Attraverso tali immagini, Gautier risale a una festa con fuochi d'artificio che sotto l'Impero aveva avuto luogo a Parigi sul Pont Royal. All'inizio dello spettacolo pirotecnico, infatti, la folla, che stazionava sul Pont des Arts, ebbe a chinarsi a tal punto sul parapetto che il ponte si sollevò e solo un rapido balzo all'indietro degli astanti evitò una terribile sciagura.

Anche a Firenze l'occhio esperto del pittore si posa sull'oggetto femminile; la ricerca gautieriana non si esaurisce, invero, su paesaggi architettonici, fontane, ponti o spazi aperti ma indaga sui volti e sulle silhouettes di dame affascinanti, siano esse fiorentine o straniere; in altre parole, il récit coglie il senso, come in un'appropriazione simbolica. E quale miglior spazio teatrale, quale più raffinato scenario di quello delle Cascine? La passeggiata alle Cascine «espèce de Champs-Elysées et de Hyde Park toscan ${ }^{63}$ era un rituale all'epoca. Il Parco delle Cascine, che si sviluppa tra l'Arno, il canale Macinante e il Mugnone, alla metà del Xvi secolo costituiva la tenuta dell'isola di proprietà dei Medici, destinata all'allevamento bovino e alle antiche fattorie che ne popolavano il territorio. Di qui il suo nome. Nel Seicento diventò orto botanico e solo all'inizio dell'Ottocento il parco fu aperto al pubblico. In mezzo a boschi popolati da pini marini, lecci, sugheri «se dessinent des chemins sablés qui aboutissent à un rond-point formant ce que les Espagnols appelleraient le salon de cette promenade fashionable» ${ }^{64}$.

E lo spettacolo ha inizio in una scenografia che l'esteta tratteggia con grande gusto e maliziosa partecipazione. Sono signore e cavalieri, dalle tenues raffinatissime e alla moda, dettagliatamente descritte in giochi di intrecci che colpiscono il lettore, al punto di poterli immaginare, percependone il décor intrigante.

La conclusione dell'ultima tappa del viaggio è ancora una volta porta alla meditazione estetica, che fa da audace supporto alla narrazione odeporica, cosicché in questo testo di rara bellezza è ancora una volta l'Art a chiudere la scena, nella descrizione della fugace visione di una dama misteriosa, non più rivista, che pareva uscita dalle pagine di un récit fantastique e che forse ispirò a Gautier la contessa polacca Prascovie Labinska in Avatar:

une apparition mystérieuse intrigua beaucoup aussi, à cette époque, la curiosité cosmopolite de Florence: une femme seule, et du plus grand air, avait paru aux Cascines, allongée dans le fond d'une calèche brune, [...] était-elle Anglaise, Italienne ou Française? C'est ce que nul ne peut résoudre, car personne ne la connaissait. Elle fit le tour des Cascines, s'arrêta un instant sur le rond-point, ne paraissait ni occupée ni surprise d'un spectacle qui semblait devoir être nouveau pour elle, et reprit le chemin de la ville ${ }^{65}$. 